\title{
Advanced Active Power Control considering the Characteristics of Distributed Energy Resources in Microgrid
}

\author{
Jin-Young Choi* and Dong-Jun Won ${ }^{\dagger}$
}

\begin{abstract}
A microgrid operator should strictly manage the distributed energy resources considering their characteristics. This paper proposes a power balancing algorithm among the distributed energy resources according to their characteristics in a microgrid. In this algorithm, a battery energy storage system is originally operated in feeder flow control mode to control the power from the utility. A supercapacitor operates through a P-f droop control to improve the power quality. Considering the low energy capacity of the super-capacitor, the battery energy storage system also restores the frequency during island operation. A distributed generator balances the energy through the microgrid central controller. The analyses of the proposed algorithm are presented and verified by PSCAD/EMTDC simulations.
\end{abstract}

Keywords: Microgrid, Distributed energy resource, Energy storage system, Distributed generation, Droop control, Restoration control, Microgrid central controller

\section{Introduction}

Distributed energy resources (DERs) have become increasing attractive due to the ever-increasing power demands on distribution networks. DERs include a wide range of electrical energy technologies such as distributed generators (DGs), renewable energy resources (RESs), and energy storage systems (ESSs). DER applications encompass power support at the substations, high fuel efficiency by capturing waste heat, the use of renewable energy, higher power quality, and smart distribution systems $[1,2]$.

A microgrid $(\mathrm{MG})$ is a future distribution network technology that is defined as a low- or medium- voltage distribution network consisting of clustered DERs and loads. An MG normally operates in parallel with the grid and allows island operation when the power quality from the grid falls below certain standards [3].

Many studies of an MG operation have been carried out, including interconnection schemes between the MG and main grid [4-7], as well as frequency and voltage control schemes within the MG during island operation.

Previously, two active power control modes using a power versus frequency droop controller in a DER control, namely, feeder flow control (FFC) mode and unit power control (UPC) mode, were presented [8]. A power balancing algorithm of a battery energy storage system (BESS) using the UPC mode was reported [9]. In addition, the power balancing algorithm of multiple DERs through each droop control mode in an MG was analyzed and verified [10].

$\dagger$ Corresponding Author: School of Electrical Engineering at INHA University, Korea. (djwon@inha.ac.kr)

* School of Electrical Engineering at INHA University, Korea (jy128308@gmail.com)

Received: February 12, 2016; Accepted: March 29, 2016
These controls are inverter-based and can change the power of a source in less than one cycle, which further improves the stability of small power systems. On the other hand, a power balancing algorithm that considers the different characteristics of DERs and restoration control of the frequency has not been reported previously.

For stable MG operation, it is essential to consider the characteristics of DERs. This paper describes the role of DERs according to their different characteristics and a power balancing algorithm of the microgrid. Section 2 presents the role of the DERs according to their characteristics. Section 3 proposes the power balancing algorithm of the microgrid, which depends on the role of the DERs.

The remainder of this paper verifies the proposed algorithm. Section 4 outlines the MG configuration and simulation model including a BESS, a super-capacitor (SC) and a DG. Section 5 presents the simulation results demonstrating the performance of the proposed power balancing algorithm, and Section 6 reports the conclusion. Detailed models of each element are summarized in the Appendix.

\section{Role of Distributed Energy Resources}

Considering the ever-increasing interest in smart grids, the developments of a variety of DERs have increased. Currently, the available DERs can be largely divided into DGs, RESs and ESSs. Each DER has different properties, such as fueling ability, energy capacity, predictability, lifetime, and ramp rate limit.

In an MG, some of the controllable power sources are required to match the momentary load fluctuations during 
Table 1. Roles of each controllable DER considering the characteristics

\begin{tabular}{c|c|c|c|c}
\hline & $\begin{array}{c}\text { Fuel } \\
\text { supply }\end{array}$ & $\begin{array}{c}\text { Energy } \\
\text { capacity }\end{array}$ & $\begin{array}{c}\text { Ramp rate } \\
\text { limit }\end{array}$ & Role \\
\hline SC & No & Low & No & - Transition Stability \\
\hline BESS & No & Middle & No & - Frequency Control \\
\hline DG & Yes & Unlimited & Yes & Energy Balancing \\
\hline
\end{tabular}

island operation. In addition, some available power sources for long term use are needed to balance the energy within the MG.

Generally, ESSs based on an inverter increase the output power instantaneously without a ramp rate limit, but they have limited energy capacity. DGs can supply the required power continuously through fueling, but they have the constraints of a ramp rate limit. RESs are unsuitable as a control source of the MG because of the large output variations and the uncertainty in prediction.

Therefore, a power balancing algorithm among the controllable DERs should be considered depending on their characteristics. Table 1 lists the roles of controllable DERs, except for RESs, according to the fueling, energy capacity and ramp rate limit.

\subsection{Roles of energy storage systems}

Normally, the ESS applications are classified as bulk energy storage for load-leveling with $10 \mathrm{MW}$ for $1-8 \mathrm{~h}$, controllable power source for peak shaving or UPS with $10-2000 \mathrm{~kW}$ for $0.5-4 \mathrm{~h}$, and power quality or end-use reliability with $100-2000 \mathrm{~kW}$ for $1-30 \mathrm{~s}$ [11]. The application known as peak shaving helps reduce both the overall energy costs of the customers by reducing the peak demand and helps increase the capacity of the main grid to serve other customers [12].

In the present study, the BESS, which has a higher energy capacity than the SC, plays an important role in shaving the peak demand and storing the surplus electrical or renewable energy through frequency control. This process is accomplished by FFC mode of the P-f droop control; hence, the MG can be considered as a controllable load from the main grid. On the other hand, SC is controlled by the UPC mode of the P-f droop control so that the transients between the island and grid-connected operation can be reduced.

Despite this, UPC mode of the SC is limited because of the low energy capacity. In this paper, a frequency restoration control within a few seconds is added to the BESS to prevent continuous discharge of the SC during island operation. The detailed control methods are described in Section 3.

\subsection{Roles of distributed generators}

Generally, DGs can enhance the overall energy efficiency of a power system and become much more cost-effective to customers within the MG under the control of a microgrid central controller (MGCC). In this study, the DG services the power with slow load variations within the MG so that the total energy is balanced. In addition, the DG can supply additional energy to the ESS when the state of charge (SOC) of the ESS is insufficient. Unlimited energy capacity is required to consistently perform these controls. The details of the control method are described in Section 3.

\section{The Power Balancing Algorithm of Microgrid}

This section describes the power balancing algorithm among the DERs considering their characteristics and analyzes the principle. In this study, the DG without a limited energy capacity performs energy balancing through the MGCC. Two ESSs with different energy capacities participate in the frequency control regarding the fast load fluctuations and the transition control by the droop controller of a micro-source controller (MC). In the ESS case, the BESS operates in FFC-mode, which keeps the feeder flow from the utility constant. The SC operates in UPC-mode so the transition stability between the islandand grid connected- operation can be improved further. In addition, it can assist the BESS during island operation. During island operation, a BESS is needed to restore the frequency due to the low energy capacity of the SC and power quality. In this study, it is assumed that the frequency of the utility is kept constant at a nominal value.

\subsection{Droop and restoration control}

BESS can offer the flexibility of supplying bi-directional power so that the positive or negative feeder flow $\left(\mathrm{P}_{\text {grid }}\right)$ relative to a flow reference is kept constant. If the amount of power imported/exported from the main grid is kept constant, the stresses in the utilities are decreased, and the main grid can easily manage the $\mathrm{MG}$ as a controllable load. In FFC mode [9], the relationship between the frequency and feeder flow can be expressed.

$$
R_{f}=\left|\frac{f_{0}-f}{P_{\text {Grid }}^{0}-P_{\text {Grid }}}\right|, P_{\text {Grid }}=P_{\text {Grid }}^{0}-\frac{1}{R_{f}}\left(f_{0}-f\right)
$$

where $R_{f}$ is the FFC droop coefficient of the BESS, $f$ and $P_{\text {Grid }}$ are the frequency and power from the main grid at a new operating point, respectively, $f_{0}$ is the nominal frequency, and $P_{\text {Grid }}^{0}$ is the initial operating point.

$$
\begin{gathered}
P_{\text {Grid }}^{\prime}=P_{\text {Grid }}^{0}-\frac{1}{R_{f}}\left(f_{0}-f^{\prime}\right)+P_{r s t}^{r e f} \\
P_{r s t}^{r e f}=\frac{1}{T_{i}} \int\left(f_{0}-f^{\prime}\right) d f
\end{gathered}
$$



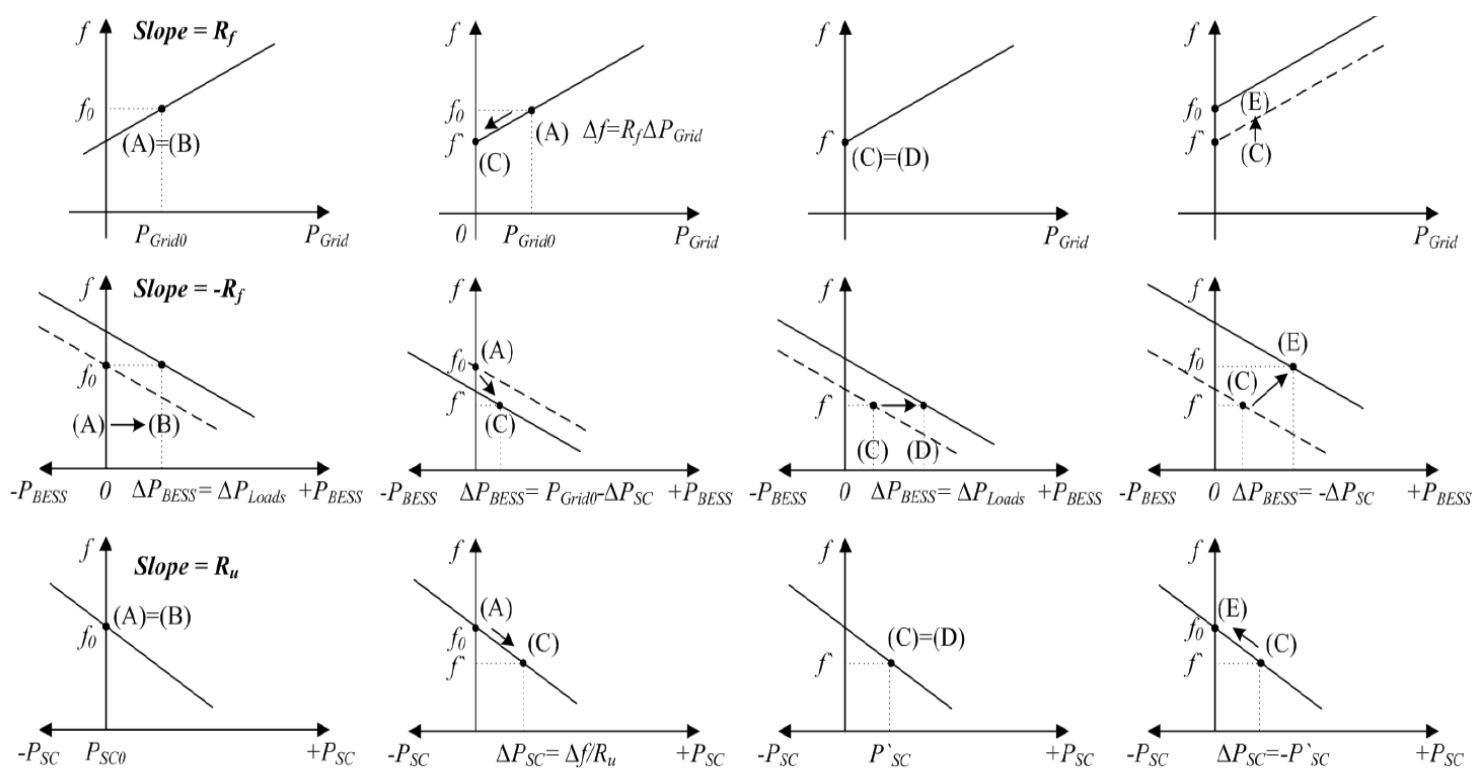

(a)

(b)

(c)

(d)

Fig. 1. Steady-state analysis of the operation points of BESS and SC according to the different droop control modes: BESS operates in FFC-mode and SC operates in UPC-mode: (a) Changing operation points of BESS and SC about the load variation during the grid-connected operations; (b) When the MG disconnects from the main grid; (c) During the island operations; (d) Restoration control of BESS during the island operations; Positive value of $P_{\text {Grid }}$ is import power from main grid and positive values of $P_{B E S S}$ and $P_{S C}$ are discharging.

During island operation, the BESS is needed to quickly restore the frequency within a few seconds to prevent full discharge of the SC due to the low energy capacity. In the frequency restoration control of the FFC mode, the restoration reference, $P_{r s t}^{r e f}$, can be added in (1) as the following (2)-(3). $T_{i}$ is the time constant of integrator of the frequency restoration control.

The SC can support the BESS operation to improve the power quality through the UPC mode of the P-f droop control in (4). Here, $R_{u}$ is the UPC droop coefficient of the $\mathrm{SC}, P_{S C}$ is the active power of the $\mathrm{SC}$ at a new operating point, and $P_{S C}^{0}$ is the initial operating point.

$$
R_{u}=-\left|\frac{f_{0}-f}{P_{S C}^{0}-P_{S C}}\right|, P_{S C}=P_{S C}^{0}-\frac{1}{R_{u}}\left(f_{0}-f\right)
$$

In UPC mode [9], the relationship between the frequency and the SC power can be expressed as (4). During the gridconnected operation, the frequency is kept constant by the utility so that the power from the main grid $\left(P_{G r i d}\right)$ can be maintained constant.

When the load increases, the BESS increases the output power from (A) to (B) to keep the $P_{\text {Grid }}$ constant, as shown in Fig. 1(a). The SC does not change the output because of the fixed frequency. The $P_{\text {Grid }}$ remains unchanged regardless of the load variations within the MG. When the MG is disconnected from the main grid, the $P_{\text {Grid }}$ drops to zero. In this case, the steady-state point of the frequency is first changed from (A) to (C) according to $R_{f}$ of the FFC
Table 2. Steady-state analysis of BESS and SC according to the transition

\begin{tabular}{c|c|c|c}
\hline & $\begin{array}{c}\text { Grid-connected } \\
\text { operation }\end{array}$ & $\begin{array}{c}\text { Disconnected } \\
\text { from main grid }\end{array}$ & $\begin{array}{c}\text { Island } \\
\text { operation }\end{array}$ \\
\hline$\Delta P_{\text {Load }}$ & $\Delta P_{\text {Load }}$ & 0 & $\Delta P_{\text {Load }}$ \\
\hline$\Delta P_{\text {Grid }}$ & 0 & $-P_{\text {Grid }}^{0}$ & 0 \\
\hline$\Delta P_{B E S S}(\mathrm{FFC})$ & $\Delta P_{\text {Load }}$ & $P_{\text {Grid }}^{0}-\Delta P_{S C}$ & $\Delta P_{\text {Load }}$ \\
\hline$\Delta P_{S C}(\mathrm{UPC})$ & 0 & $-\Delta f / R_{u}$ & 0 \\
\hline$\Delta f$ & 0 & $\Delta P_{\text {Grid }} \times R_{f}$ & 0 \\
\hline
\end{tabular}

mode of the BESS, as shown in Fig. 1(b). Subsequently, the BESS increases the output power to compensate for the decreased feeder flow, $P_{\text {Grid }}$. The SC increases the out power according to $R_{u}$ because of the UPC mode of the P-f droop control by the frequency variation. The frequency of the islanded MG is determined at a new operating point, where the sums of the output power of BESS and SC are equal to $P_{\text {Grid }}$.

During island operation, $P_{\text {Grid }}$ is always zero, and the frequency does not change, even if the load fluctuates, which means that the output of the BESS and SC can be explained in a similar manner to that of the grid-connected operation, as shown in Fig. 1(c). The frequency of the MG is restored to the normal value, $\mathrm{f}_{0}$, by frequency restoration of the BESS so that the SC decreases the output from (C) to $(\mathrm{E})$, and the BESS compensates for the decreased output power of the SC, as shown in Fig. 1(d). Table 2 lists the results according to the different droop controls of the 
BESS and SC.

\subsection{MGCC control}

Typically, an MGCC with communication networks manages and monitors all elements to improve the reliability, stability, and economics of an MG. In this study, the MGCC performs energy balancing within the MG and commends the output operating point of each element $\left(P_{\text {Grid }}^{0}, P_{S C}^{0}, P_{D G}^{0}\right)$ through the following procedures.

Step 1) The MGCC estimates the required total loads. Here, the output of the RESs is regarded as a negative load. This process is accomplished by adding the active power of the BESS, SC and DG to the network system.

$$
P_{\text {Load }}^{\text {est }}=P_{D G}+P_{B E S S}+P_{S C}
$$

Step 2) The MGCC commends the slow dynamics reference $P_{\text {Load }}^{\text {Slow }}$ of the total load to the DG using a low pass filter considering the ramp rate limit. In addition, the operating point of the DG is limited by the maximum and minimum output, $\left(P_{D G}^{\max }, P_{D G}^{\min }\right)$.

$$
P_{D G}^{0}=\left(\begin{array}{ll}
P_{D G}^{\max } & \left(P_{\text {Lood }}^{\text {Slow }} \geq P_{D G}^{\max }\right) \\
P_{\text {Load }}^{\text {Slow }} & \left(P_{D G}^{\min } \leq P_{\text {Load }}^{\text {Slow }} \leq P_{D G}^{\max }\right) \\
P_{D G}^{\min } & \left(P_{\text {Load }}^{\text {Slow }} \leq P_{D G}^{\min }\right)
\end{array}\right)
$$

Step 3) The MGCC determines the initial operating point to manage the energy of the ESSs. When the ESS has been reached without the SOC limits, the FFC mode of the BESS should have changed to UPC mode to manage the energy stably [13]. In this case, the operating point of the UPC mode $\left(P_{B E S S}^{U P C}\right)$ is expressed as (7).

$$
\begin{gathered}
\text { If }\left(S O C_{i}>S O C_{i}^{\max }\right) \\
\text { While }\left(S O C_{i} \geq 0.5\right), P_{i}^{U P C}=\alpha P_{i}^{\text {rate }} ; \\
\text { If }\left(S O C_{i}<S O C_{i}^{\text {min }}\right) \\
\text { While }\left(S O C_{i} \leq 0.5\right), P_{i}^{U P C}=-\alpha P_{i}^{\text {rate }} ;
\end{gathered}
$$

where $i$ is the BESS or SC, a positive value of $P_{i}^{U P C}$ denotes the discharged power, and a negative value denotes the charged power. $P_{i}^{\text {rate }}$ is the rated power of BESS or SC. $\alpha$ was set from zero to one by the operator.

Step 4) If the load is much higher than the sum of the capacities of all DERs, the MG should perform load shedding.

\section{Microgrid Configuration and Control System}

To verify the proposed control, the MG is comprised of a FC with a ramp rate limit, one type of DGs, and two ESSs

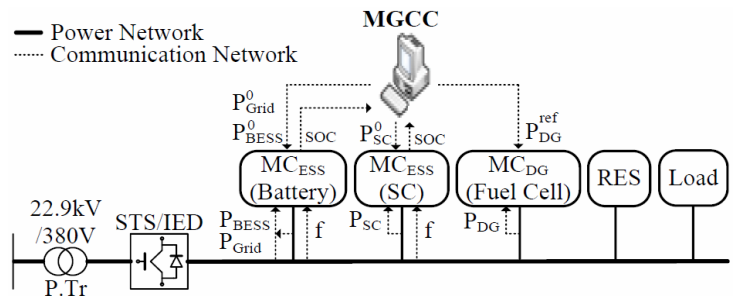

Fig. 2. Microgrid configuration and control system

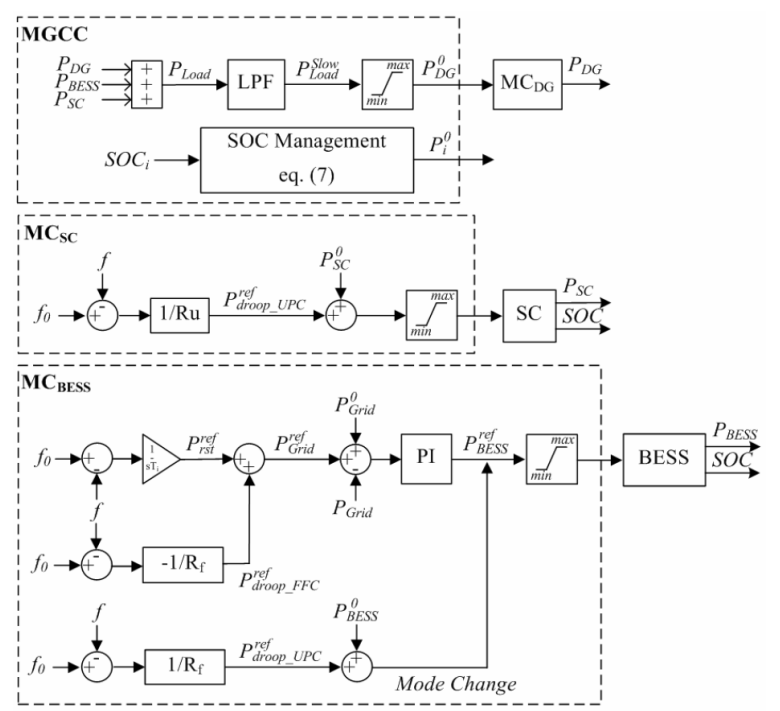

Fig. 3. Block diagram of proposed controllers: MCs of ESSs and a MGCC

with different limited energy capacities, such as the BESS and SC. In addition, clustered loads, which include renewable energy sources, are connected to the $\mathrm{MG}$, as shown in Fig. 2.

Through the MGCC and MCs with a network system, the controllable DERs are regulated according to their roles Fig. 3 presents the proposed controllers of the MGCC and MCs.

\section{Case Study}

To verify the proposed control algorithm, a $50 \mathrm{~kW} /$ $50 \mathrm{kWh}$ lithium-ion battery, a $50 \mathrm{~kW} / 500 \mathrm{kWs}$ SC and a 100 $\mathrm{kW}$ proton exchange membrane fuel cell (PEMFC) were used, and the simulation was performed using PSCAD/ EMTDC. The BESS parameters were set to the following: $R_{f}=0.01, P_{\text {Grid }}^{0}=15 \mathrm{~kW}(0.3 \mathrm{pu})$. The SC parameters were set to the following: $R_{u}=-0.0067, P_{S C}^{0}=0 \mathrm{~kW}(0.0 \mathrm{pu})$. The cutoff frequency of the low pass filter of the MGCC was $0.3 \mathrm{~Hz}$ and $\alpha=0.5$. The minimum output of the PEMFC was $10 \mathrm{~kW}(0.1 \mathrm{pu})$

\subsection{Case A : FFC mode of BESS and UPC mode of SC}

In case $\mathrm{A}$, a BESS and a $\mathrm{SC}$ without a $\mathrm{DG}$ operate 


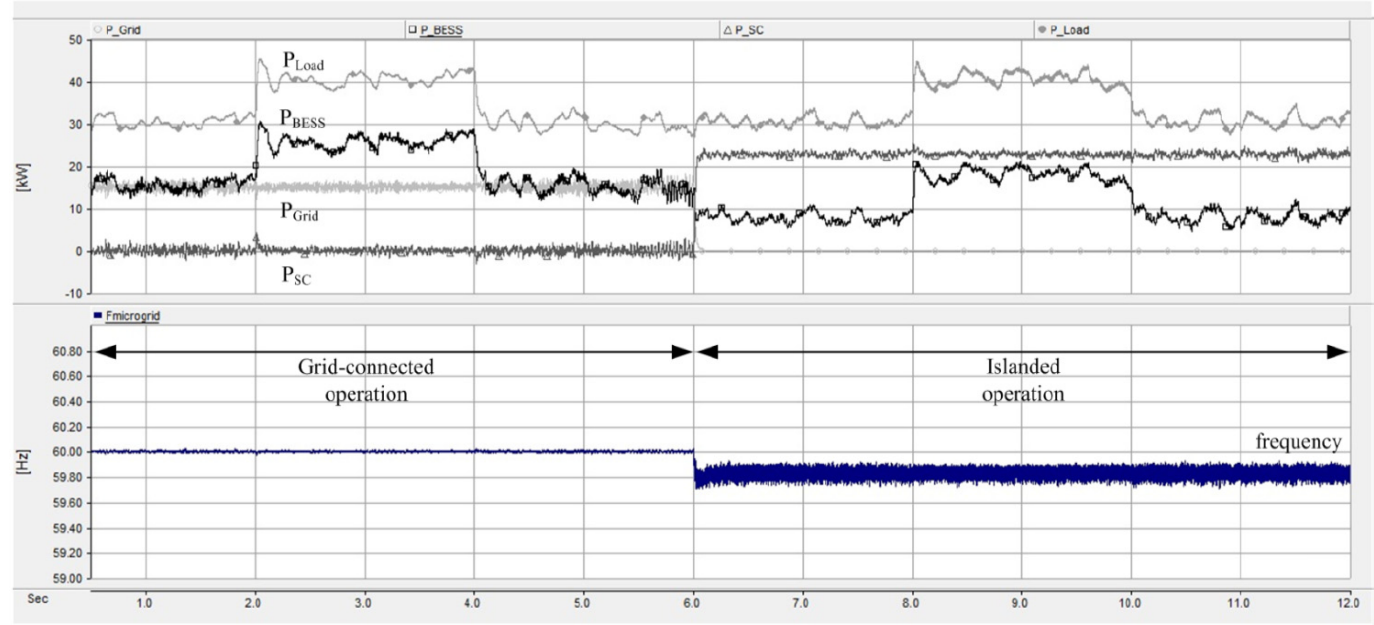

Fig. 4. Simulation result of case A: BESS - FFC mode of P-f droop, SC - UPC mode of P-f droop

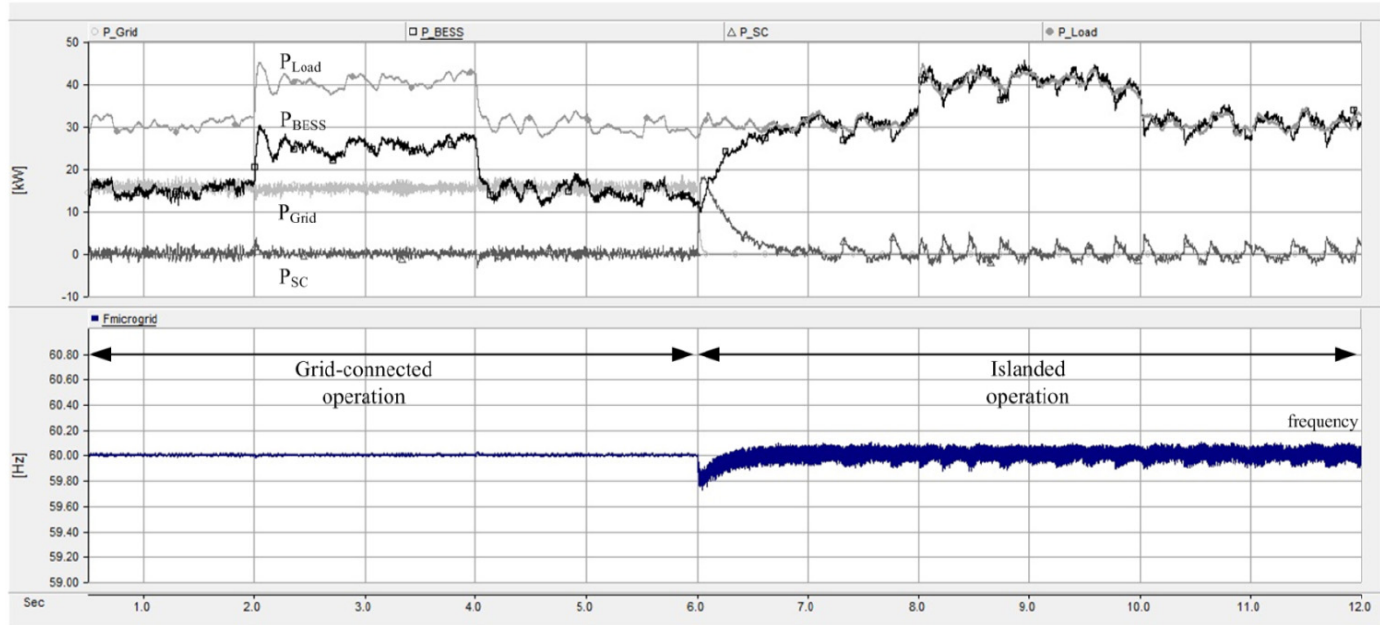

Fig. 5. Simulation result of case B: BESS - FFC mode of P-f droop and frequency restoration, SC - UPC mode of P-f droop

together through droop control, as shown in Fig. 4. During the grid-connected operation (0-6s), PBESS changes quickly according to the load variation by FFC mode, which operates the point changes from (A) to (B), as shown in Fig. 1(a). At that time, the $P_{S C}$ and $P_{\text {Grid }}$ are kept constant because of the constant frequency.

When the MG is disconnected from the main grid (6s), the BESS and SC are operated by P-f droop control, as shown in Fig. 1(b). Therefore, the operating points are $f$ $=59.82 \mathrm{~Hz}, P_{S C}^{\prime}=22.38 \mathrm{~kW}$, and $P_{B E S S}^{\prime}=7.62 \mathrm{~kW}$.

During island operation (6-12s), the frequency of MG is kept constant by FFC-mode of the BESS, and the SC produces a constant active power. Therefore, BESS takes charge of the lack of or surplus of power, as shown in Fig. 1(c).

\subsection{Case B : FFC mode and frequency restoration of BESS}

A potential risk factor exists when the ESS continuously discharges or charges because of the energy capacity factor. The SC will have the low energy capacity within a few seconds or minutes. If the BESS accomplishes the proposed the frequency restoration control in a few seconds, the SC can operate stably within the limits of the energy capacity, as shown in Fig. 1(d).

Fig. 5 shows that the frequency of the MG is restored to the nominal value $(60 \mathrm{~Hz})$ by restoration control of the BESS during island operation. Hence, the BESS takes charge of most of the load variations, and the SC quickly compensates for the instantaneous variation; thus, the transient stability of the MG is improved.

\subsection{Case C : MGCC control of DG}

In case $\mathrm{C}$, an additional $\mathrm{FC}$ takes charge of the slow load variations through MGCC, as shown in Fig. 6. The energy balance within the MG is kept for a long time by the FC. Therefore, the burden of the BESS can be eased more than in cases $\mathrm{A}$ and $\mathrm{B}$, which means that robust performance is 


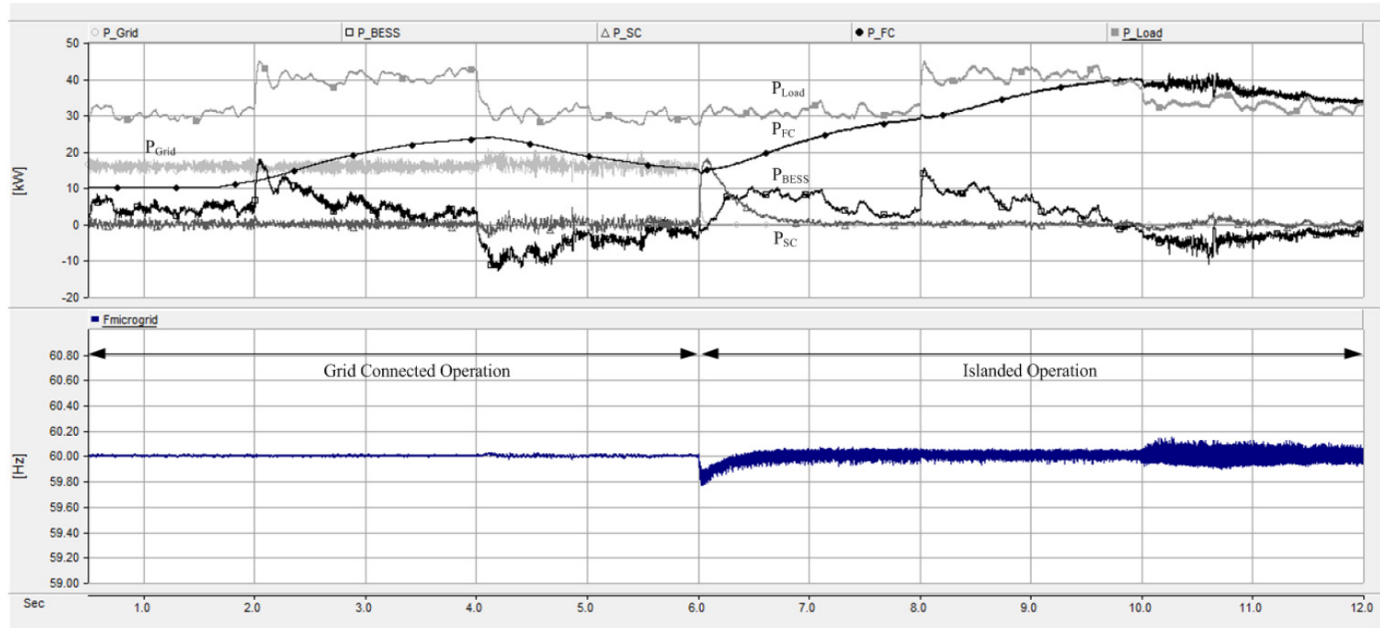

Fig. 6. Simulation result of case C: BESS - FFC mode of P-f droop and frequency restoration, SC - UPC mode of P-f droop, FC - MGCC control

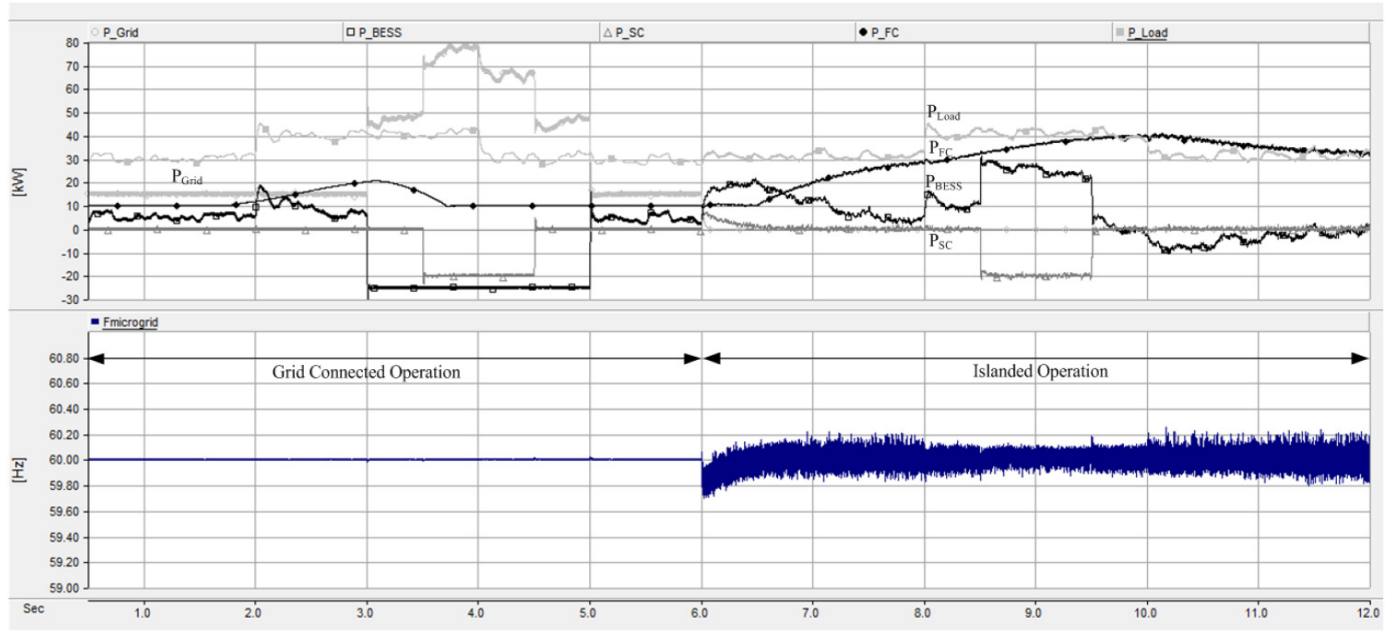

Fig. 7. Simulation result of case D: BESS - control mode change (FFC/UPC) to $3 \sim 5 \mathrm{sec}$, SC - operation point change to 3.5 4.5sec, FC - MGCC control

improved during the transient.

\subsection{Case D : MGCC control of ESS}

Fig. 7 shows that the MGCC provides the control mode of the BESS and the operating points of each ESS when their SOCs are reached without the limits. The BESS changes from FFC mode ( $P_{\text {Grid }}^{0}=15 \mathrm{~kW}$ ) to UPC mode $\left(P_{B E S S}^{U P C}=25 \mathrm{~kW}\right)$ in $3-5 \mathrm{~s}$. The operating point of the SC also changes from $P_{S C}^{0}=0 \mathrm{~kW}$ to $P_{S C}^{0}=15 \mathrm{~kW}$ in $3.5 \sim 4.5 \mathrm{~s}$. The FC output decreases by $P_{\text {Load }}^{\text {est }}$ and is limited to the minimum output. Therefore, the power from the main grid $\left(P_{\text {Grid }}\right)$ takes charge of the lack power.

After $5 \mathrm{~s}$, the $P_{\text {Grid }}$ is kept to a set value $\left(P_{\text {Grid }}^{0}=15 \mathrm{~kW}\right)$ by the FFC mode of the BESS. During island operation, the SC changes from $P_{\text {Grid }}^{0}=0 \mathrm{~kW}$ to $P_{\text {Grid }}^{0}=15 \mathrm{~kW}$ in $8.5 \sim 9.5 \mathrm{~s}$. The BESS and FC are under a similar situation to that in case $\mathrm{C}$.

\section{Conclusion}

For stable operation of the MG, it is essentail to consider the characteristics of the DERs, such as fueling, ramp rate limit, and energy capacity. A power balancing algorithm among the various DERs within the MG has been presented. In the proposed power balancing algorithm, the role of the DERs is established clearly according to their characteristics.

The DG with a ramp rate limit performs energy balancing on the slow variations in the load through the MGCC. The ESSs with a droop controller could automatically match the fast variations in the load within their capacity limits during the grid-connected and island operations. Through the MGCC, the SOCs of the ESSs are kept properly within the limits of their energy capacities.

The BESS with a high energy capacity and without a ramp rate limit operates in FFC mode of the P-f droop control so that the power supplied from the main grid is 
kept constant regardless of the load variations in the MG. Therefore, the MG is considered as a controllable load from a utility point of view, which facilitates participation in the demand response market and provides more profit.

The SC with a low energy capacity and without a ramp rate limit operates in UPC mode of the P-f droop control so that the transient between the island and grid-connected operations can be reduced, which improves the MG power quality. On the other hand, the UPC mode of the SC is limited because of the low energy capacity. In this study, frequency restoration control within a few seconds is added to the BESS to prevent continuous discharge of the SC during island operation.

The proposed scheme provides an appropriate and stable power balancing algorithm among the various DERs by considering their characteristics. The MG can effectively manage the DERs and obtain more profit by keeping constant the power supplied by the utility. The simulation suggests that the DERs share the appropriate amount of power according to each role, and the system stability is maintained within acceptable limits.

\section{Appendix}

\subsection{Modeling of battery energy storage system}

In its most basic form, the Thevenin-based model of the BESS uses a series resistor $\left(\mathrm{R}_{\mathrm{S}}\right)$ and a $\mathrm{RC}$ parallel network $\left(\mathrm{R}_{\mathrm{T}}\right.$ and $\left.\mathrm{C}_{\mathrm{T}}\right)$ to predict the battery response to transient load events. Many studies have examined the modeling of BESS that considers the transient characteristics, SOC, and lifetime $[14,15]$. The BESS, which is a simplified model proposed in [15], was adopted in the present study, and the capacity was modified to $50 \mathrm{~kW} / 50 \mathrm{kWh}$. The extracted nonlinear open-circuit voltage $\mathrm{V}_{\mathrm{OC}}\left(\mathrm{V}_{\mathrm{SOC}}\right), \mathrm{R}_{\mathrm{S}}$, and $\mathrm{RC}$ network $\left(\mathrm{R}_{\mathrm{T} \_\mathrm{S}}, \mathrm{C}_{\mathrm{T} \_\mathrm{S}}, \mathrm{R}_{\mathrm{T} \_\mathrm{L}}, \mathrm{C}_{\mathrm{T} \_\mathrm{L}}\right)$ are as follows:

$$
\begin{aligned}
& V_{O C}(S O C)=-1.031 \cdot e^{-35 \cdot S O C}+3.685+0.2156 \cdot S O C \\
& -0.1178 \cdot S O C^{2}+0.3201 \cdot S O C^{3} \\
& R_{S}(S O C)=0.1562 \cdot e^{-24.36 \cdot S O C}+0.07446 \\
& R_{T_{-} S}(S O C)=0.3208 \cdot e^{-29.14 \cdot S O C}+0.04669 \\
& C_{T_{-} S}(S O C)=-752.9 \cdot e^{-13.51 \cdot S O C}+703.6 \\
& R_{T_{-} L}(S O C)=6.603 \cdot e^{-155.2 \cdot S O C}+0.04984 \\
& C_{T_{-} L}(S O C)=-6056 \cdot e^{-27.12 \cdot S O C}+4475
\end{aligned}
$$

This equivalent model is interfaced with a bi-directional DC/DC converter, which maintains a constant DC-link voltage. A DC/AC inverter, which controls the output power using the proposed FFC mode and frequency restoration controller, is connected to the MG.

\subsection{Modeling of super-capacitor}

The equivalent model of the SC can generally be simplified to an ideal capacitor $\mathrm{C}$ and an equivalent series resistance $R_{S C}$, where the $C_{S C}$ is large but the $R_{S C}$ is very small [16-17]. In this study, the $\mathrm{SC}$ parameters $(50 \mathrm{~kW} /$ $500 \mathrm{kWs}$ ) were set to $\mathrm{C}_{\mathrm{SC}}=10.09 \mathrm{~F}$ and $\mathrm{R}_{\mathrm{SC}}=0.0781 \mathrm{ohm}$ using the reference data [18]. This equivalent model was interfaced with a bi-directional DC/DC converter similar to that in the BESS. A DC/AC inverter, which controls the output power by the UPC-mode of the P-f droop control, was connected to the MG.

\subsection{Modeling of distributed generator}

Many studies have modeled the PEMFC. An equivalent electrical circuit of the double-layer charging effect inside the PEMFC has been proposed [19]. This PEMFC model, as a DG, was adopted in this study, where the capacity was modified to $50 \mathrm{~kW}$. This equivalent model was interfaced with a boost-buck converter, which keeps the DC-link voltage constant. A DC/AC inverter, which controls the output to the reference power of the MGCC, was connected to the MG.

\subsection{Modeling of load}

All simulation scenarios used a fixed PQ load and a variable load, which were interfaced with a DC/AC inverter as a renewable energy source.

\section{Acknowledgements}

This work was supported by an INHA University Research Grant. And this work was supported by Basic Science Research Program through the National Research Foundation of Korea (NRF) funded by the Ministry of Education, Science and Technology (grant number: NRF2013R1A1A1012667).

\section{References}

[1] Rovert H.Lasseter, "Smart Distribution: Coupled Microgrids", Proceedings of the IEEE, vol. 99, no. 6, pp. 1074-1082, June. 2011.

[2] S.schowdhury; et al, "Microgrids and Active Distribution Networks", IET Press, 2009. pp. 57-76.

[3] Alvaro Llaria, et al, "Survey on microgrids: Unplanned islanding and related inverter control techniques", Renewable Energy, Vol. 36, no. 8, pp. 2052-2061, Aug. 2011.

[4] F. Katiraei, M. R. Iravani, Fellow, "Power Management Strategies for a Microgrid With Multiple Distributed Generation Units", IEEE Trans. on Power Systems, vol. 21, no. 4, Nov. 2006.

[5] De Brabandere, et al, "A Voltage and Frequency Droop Control Method for Parallel Inverters", IEEE 
Trans. on POWER ELECTRONICS, Vol. 22, no. 4, pp. 1107-1115, July 2007.

[6] Seon-Ju; Seung-Il Moon, et al, "Power-Sharing Method of Multiple Distributed Generators considering Control Modes and Configurations of a Microgrid", IEEE Trans. on POWER DELIVERY, Vol. 25, no. 3, pp. 2007-2016, July 2010.

[7] Loc Nguyen Khanh, "Control Strategies for DGs to Improve Operation Performance of Microgrid", Dissertation of Inha University, Aug. 2011.

[8] P. Piagi, R. H. Lasseter, "Autonomous control of microgrids", presented at the Power Eng. Soc. General Meeting, Montreal, QC, Canada, 2006.

[9] Rovert H.Lasseter,et al, "Integration of Distributed Energy Resources The CERTS Microgrid Concept", California energy commission, California, Rep. P500-03-089F, Oct. 2003.

[10] E. Barklund, et al, "Energy Management in Autonomous Microgrid Using Stability-Constrained Droop Control of Inverters", IEEE Tran. on Energy Conversion, vol. 19, no. 1, March 2008.

[11] Susan M. Schoenung, "Characteristics and Technologies for Long-vs. Short-Term Energy Storage", Sandia National Laboratories, SAND2001-0765, pp. 2-3, March, 2001.

[12] Y.Zhu and K. Tomsovic, "Development of models for analyzing the load-following performance of microturbines and fuel cells", Electric Power Systems Research, vol. 62, pp. 1-11, 2002

[13] Jin-Young Choi, Dong-Jun Won, "Modified Feeder Flow Control Method of BESS using Droop Control in Microgrid", International Exhibition and Conference for PCIM, June, 2012.

[14] D. Linden and T. B. Reddy, "Handbook of Batteries", McGraw-Hill Press, 3rd ed., Chapter 5 and Chapter 14, 2002.

[15] Min Chen, el al, "Accurate Electrical Battery Model Capable of Predicting Runtime and I-V performance", IEEE Trans. on Energy Conversion, Vol. 21, no. 2, pp. 504-511, 2006.

[16] Tang Xisheng, "Research on Energy Management and Stability of Distributed Generation System with EDLC as Energy Storage", Beijing: Chinese Academy of Sciences, 2006.

[17] Wu Bingbing, et al, "Super-capacitors Energy Storage System Applied in the Microgrid", ICIEA, 2010 the 5th IEEE Conference on, pp. 1002-1005, 2010.

[18] http://www.nesscap.com/product/module.jsp

[19] Caisheng Wang, et al, "Dynamics Models and Model Validation for PEM Fuel Cells using Electrical Circuits", IEEE Trans. on ENERGY CONVERSION, Vol. 20, no. 2, June 2005.

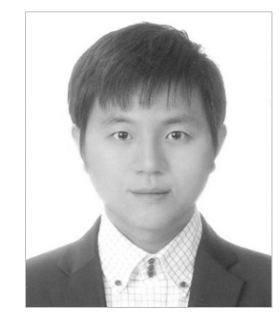

Jin-Young Choi He received the B.S., M.S. degree in electrical engineering from INHA University, Korea, in 2010, 2012. Currently, he is a Ph.D. candidate at INHA University. His research interests include smart grid, microgrid, battery energy storage system.

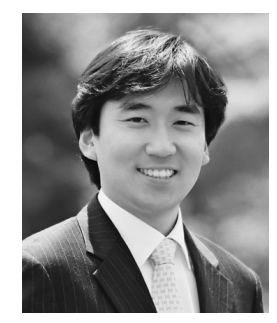

Dong-Jun Won He received B.S., M.S., and Ph.D. degrees in Electrical Engineering, Seoul National University, Seoul, Korea in 1998, 2000, and 2004 respectively. He was a Postdoctoral Fellow in the APT Center, University of Washington, Seattle. Currently, he is an Associate professor in the School of Electrical Engineering, INHA University, Korea. 